gr-qc/yymm, CGPG-94/4-4

\title{
Bases of Wormholes in Quantum Cosmology
}

\author{
Guillermo A. Mena Marugán \\ Center for Gravitational Physics and Geometry, \\ Pennsylvania State University, University Park, PA 16802, USA. \\ On leave from: Instituto de Matemáticas y Física Fundamental, \\ C.S.I.C., Serrano 121, 28006 Madrid, Spain.
}

March, 1994

\begin{abstract}
We show that if the space of physical states spanned by the wormhole wave functions can be equipped with a Hilbert structure, such a Hilbert space must coincide with that of the Lorentzian gravitational system under consideration. The physical inner product can then be determined by imposing a set of Lorentzian reality conditions. The Hilbert space of the gravitational model admits in this case a basis of wormhole solutions, and every proper quantum state can be interpreted as a superposition of wormholes. We also argue that the wave functions that form the basis of wormholes must be eigenfunctions of a complete set of compatible observables. The associated eigenvalues provide a set of well-defined wormhole parameters, in the sense that they can be employed to designate the different elements of the basis of wormholes. We analyse in detail the case of a Friedmann-Robertson-Walker spacetime minimally coupled to a massless scalar field. For this minisuperspace, we prove the validity of all the above statements and discuss various admissible choices of bases of wormhole wave functions.
\end{abstract}




\section{Introduction}

Wormholes are solutions to the equations of motion of Euclidean General Relativity that connect two asymptotic three-manifolds of large volume $[1,2]$. They can be interpreted as closed universes that branch off, or join onto, the asymptotic flat regions of spacetime [1]. It has been argued that the existence of wormholes may significantly affect the effective low-energy interactions, altering the value of the physical constants [1] and providing a mechanism for the vanishing of the cosmological constant [3].

If wormholes are to play a crucial role in physics, they should occur regardless of the specific properties of the matter content of the Universe. However, classical solutions of the wormhole type can exist only for very special kinds of matter fields, namely, those which allow the Ricci tensor to have negative eigenvalues [4]. None the less, if it is possible to construct a quantum theory of gravity, wormholes do not need to be realized as classical solutions to the Einstein equations [5]. Quantum mechanically, a wormhole will simply be a physical state (wave function) with a characteristic asymptotic behaviour in the limit of large three-geometries, such that it can represent a tube connected to a region of spacetime with large three-volume [5]. These considerations led Hawking and Page to propose that the wormhole wave functions should be exponentially damped for large three-geometries, and regular for degenerate ones [5]. The last requirement guarantees that the wave functions present no singularities when the three-geometry collapses to zero because of an ill-defined slicing of the spacetime. These wormhole boundary conditions have also been reformulated in a language that is specially well-suited for the Euclidean path integral approach to Quantum Cosmology. According to Garay [6], the wormhole wave functions can thus be represented as path integrals over Euclidean manifolds that match asymptotic large three-geometries with no gravitational excitations, with 
the gravitational momenta fixed in that asymptotic region. In particular, the wormhole ground state is obtained by demanding that, in addition, there exist no matter excitations in the regions of large volume.

In order to determine the effect of wormholes in the low-energy physics, it has been assumed in the literature that the vector space spanned by the wormhole wave functions can be equipped with a Hilbert structure [1,3]. Although this conjecture has never been proved in full gravity, its validity has been vindicated at least in a collection of minisuperspace models in which the complete set of wormhole states is known [5-8]. In all these minisuperspaces, nevertheless, the inner product in the space of wormholes has been constructed by restricting to the space of physical states an inner product that is naturally defined in the unconstrained gravitational models [7]. This proposal to fix the inner product must not be expected to work satisfactorily in more general cases, because the physical states of a constrained system need not be normalizable with respect to any pre-Hilbert structure introduced before imposing the first-class constraints of the theory. It therefore seems necessary to adopt a different prescription to determine a unique inner product in the space of wormhole wave functions.

In the last years, Ashtekar has developed a detailed programme to achieve the non-perturbative quantization of systems with first-class constraints, including the determination of the physical inner product [9-11]. One must first select a (possibly over-)complete set of complex functions on phase space that is closed under Poisson brackets, and promote it to an abstract *-algebra of operators, with the classical Poisson brackets translated into commutators and the complex conjugation relations between classical variables captured in the $*$-involution $[9,10]$. This $*$-algebra is represented on a chosen vector space, with the physical states provided by the kernel of all the operators that represent the first-class constraints of the theory. Finally, one must find a sufficiently large number of observables, i.e., operators that commute 
with all the constraints. The physical inner product is then uniquely fixed [12] by imposing the $*$-relations between observables as adjointness conditions $[9,10]$. These requirements are usually called reality conditions $[9,13]$.

We can apply this systematic programme to those minisuperspaces models for which the wormhole quantum solutions have been explicitly found, and discuss in this way whether it is possible to arrive at a well-defined Hilbert structure for the space of wormholes. Nevertheless, let us notice first that the quantization approach proposed by Ashtekar determines completely the Hilbert space of physical states once one has specified a set of reality conditions and chosen a particular representation (what includes in turn the selection of an algebra of elementary operators). Suppose now that, for a given representation, the space of wormholes admits a Hilbert structure, selected by a certain set of reality conditions. We are then led to conclude that either the reality conditions that correspond to the wormhole states are not Lorentzian, or the wormhole wave functions must span a subspace of the physical (Lorentzian) Hilbert space of the analysed quantum theory.

At first glance, one might think that, instead of being Lorentzian, the reality conditions for wormholes should be those of Euclidean gravity, because the wormhole wave functions can be represented (at least in some models) as Euclidean path integrals [6]. However, the Euclidean action that provides the weight of the different paths in those integrals can be related to the Lorentzian gravitational action by means of a Wick rotation, and we know that reality conditions are left invariant under such rotations [14]. Hence, we should actually expect that the reality conditions for the wormhole states are those corresponding to the Lorentzian theory. Note in this sense that the wormhole wave functions satisfy in fact the Lorentzian Wheeler-DeWitt equation, and not its Euclidean counterpart (i.e., the equation obtained from the Euclidean Hamiltonian constraint assuming the same commutation relations for Euclidean as for Lorentzian gravity [14]). On the other hand, in 
those minisuperspaces in which the wormhole solutions have been shown to form a Hilbert space (and even if we do not agree with the prescription adopted to select the Hilbert structure), the inner product was found by demanding that the Lorentzian momenta of the gravitational and matter fields were self-adjoint [7], requirements that can clearly be interpreted as Lorentzian reality conditions for the corresponding unconstrained gravitational models. We thus claim that the Hilbert structure of the space of wormholes must be determined by imposing an appropriate set of Lorentzian reality conditions.

As a consequence, we conclude that if for a given representation there exists a Hilbert space of wormholes, it must be a subspace of the Hilbert space of the Lorentzian quantum theory. Moreover, if we consider only irreducible representations (and assume that the space of wormholes is stable under the action of the observables of the system), the wormhole wave functions have to span the whole Hilbert space of physical states in the constructed quantization. This means that the wormhole solutions provide in this case a basis for the Hilbert space of the Lorentzian theory, and that the so-called Hilbert space of wormholes $[5,7]$ is just the physical Hilbert space of the analysed gravitational model. Notice that, in general, the wormhole wave functions that form a particular basis for the Hilbert space need not be normalizable with respect to the physical inner product, although it may be possible to find a change to a new basis of quantum states that have finite norm. In any of these bases, the different wave functions will be designated by a set of wormhole parameters $[1,3]$ which, in order to characterize the wormhole states, must possess a well-defined meaning in the constrained gravitational theory and must therefore correspond to eigenvalues of observables of the system.

In the rest of this work, we will show with an explicit example that the Hilbert space of wormholes is nothing else but the Hilbert space of the Lorentzian quantum theory. We will study the case of a Friedmann-Robertson-Walker model in the 
presence of a minimally coupled massless scalar field. For this minisuperspace, the complete set of wormhole states is known [5]. In section 2 we introduce the model and carry out its non-perturbative canonical quantization according to Ashtekar's programme, obtaining in this way the physical inner product. We then find in section 3 the integral transform that leads from the representation used in this non-perturbative quantization to the geometrodynamic representation in which the wormhole wave functions were originally found. We also prove there that the Hilbert space of the model is spanned by a basis of wormholes, and discuss the normalizabil-

ity properties of the wormhole states which form that basis. In section 4 we analyse in detail other admissible choices for the basis of wormholes. In particular, we show that there exists a discrete basis of wormhole wave functions that are normalizable with respect to the Lorentzian inner product. Finally, we present our conclusions and some further remarks in section 5 .

\section{Minisuperspace Model: Quantization}

We will consider a Friedmann-Robertson-Walker spacetime, described by the metric

$$
d s^{2}=\frac{2 G}{3 \pi}\left(-\frac{N^{2}(t)}{2} q^{3}(t) d t^{2}+2 q(t) d \Omega_{3}^{2}\right), \quad q(t)>0 .
$$

Here, $q(t)$ is the square of the scale factor of the model, $N(t)$ is the rescaled lapse function and $d \Omega_{3}^{2}$ is the metric of the unit three-sphere. The matter content of the system will consist of a minimally coupled massless scalar field, $\Phi$, that will be assumed to be homogeneous. Rescaling the scalar field as

$$
\Phi(t)=\sqrt{\frac{3}{16 \pi G}} \phi(t)
$$

the Hamiltonian constraint of the model can be expressed $[5,6]$ :

$$
H \equiv \frac{1}{2}\left(q^{2} \Pi_{q}^{2}+q^{2}-\Pi_{\phi}^{2}\right)=0, \quad q>0
$$

with $\Pi_{q}$ and $\Pi_{\phi}$ the momenta canonically conjugate to $q$ and $\phi$, respectively. 
Let us introduce now the following canonical transformation of variables [15]:

$$
q=\frac{\Pi}{\cosh X}, \quad q \Pi_{q}=-\Pi \tanh X .
$$

A generating function for this transformation is provided by

$$
F(q, X)=-q \sinh X \text {. }
$$

The first equation in (4) implies that the momentum $\Pi$ conjugate to $X$ is strictly positive, because $q>0$. The configuration variable $X$, on the other hand, runs over the whole real axis, $X \in \mathbb{R}$. Employing relations (4), the Hamiltonian constraint (3) can be rewritten as

$$
H \equiv \frac{1}{2}\left(\Pi^{2}-\Pi_{\phi}^{2}\right)=0, \quad \Pi>0 .
$$

This is the only constraint of the system, since we have already fixed the diffeomorphism gauge freedom.

Equation (6) is satisfied only if $\Pi_{\phi}=\Pi>0$ or $\Pi_{\phi}=-\Pi<0$. As a consequence, the reduced phase space of the model splits into two disconnected pieces, i.e., the cotangent bundles over the positive $\left(\Pi_{\phi}>0\right)$ and the negative $\left(\Pi_{\phi}<0\right)$ real axis. In fact, both sectors $\left(\Pi_{\phi}>0\right.$ and $\left.\Pi_{\phi}<0\right)$ are related by a time reversal operation, so that they can be considered as physically equivalent [15]. Owing to this fact, we will restrict our attention in the following to the case $\Pi_{\phi}>0$, unless explicitly stated.

To construct the quantum theory, let us choose as representation space the space of complex functions $\Psi\left(X, \Pi_{\phi}\right)$ on $\mathbb{R} \times \mathbb{R}^{+}$, with $X \in \mathbb{R}$ and $\Pi_{\phi} \in \mathbb{R}^{+}$. In this representation (and taking $\hbar=1$ from now on), we can introduce the following canonical set of basic operators:

$$
\begin{gathered}
\hat{\Pi}_{\phi} \Psi=\Pi_{\phi} \Psi\left(X, \Pi_{\phi}\right), \quad \hat{\phi} \Psi=i \partial_{\Pi_{\phi}} \Psi\left(X, \Pi_{\phi}\right), \\
\hat{X} \Psi=X \Psi\left(X, \Pi_{\phi}\right), \quad \hat{\Pi} \Psi=-i \partial_{X} \Psi\left(X, \Pi_{\phi}\right) .
\end{gathered}
$$


A simple calculation shows then that the physical states that satisfy

$$
\hat{\Pi} \Psi\left(X, \Pi_{\phi}\right)=\Pi_{\phi} \Psi\left(X, \Pi_{\phi}\right),
$$

and thus the quantum version of the Hamiltonian constraint (6), adopt the generic expression

$$
\Psi\left(X, \Pi_{\phi}\right)=f\left(\Pi_{\phi}\right) e^{i \Pi_{\phi} X},
$$

$f\left(\Pi_{\phi}\right)$ being any complex function on $\mathbb{R}^{+}$.

A complete set of classical observables on the reduced phase space of the model (the cotangent bundle over $\mathbb{R}^{+}$) is provided by $\Pi_{\phi}$ and [15]

$$
w=\Pi_{\phi} \phi+\Pi_{\phi}^{2} \frac{X}{\Pi} .
$$

This set is closed under Poisson brackets, for $\left\{w, \Pi_{\phi}\right\}=\Pi_{\phi}$. In the quantum theory, the action of these observables on the physical states (10) will be given by the first equation in (7) and

$$
\hat{w}\left(f\left(\Pi_{\phi}\right) e^{i \Pi_{\phi} X}\right)=i \Pi_{\phi}\left(\partial_{\Pi_{\phi}} f\left(\Pi_{\phi}\right)\right) e^{i \Pi_{\phi} X} .
$$

This formula can be obtained from (7-9) and the definition of $w(11)$ with an adequate choice of factor ordering.

For Lorentzian gravity, the classical variables $\Pi_{\phi}$ and $w$ are real. Therefore, the reality conditions associated with the Lorentzian theory demand the hermiticity of the operators $\hat{\Pi}_{\phi}$ and $\hat{w}$ on the space of physical states. These requirements select the inner product

$$
<\Theta, \Psi>=\int_{\mathbb{R}^{+}} \frac{d \Pi_{\phi}}{\Pi_{\phi}} g^{*}\left(\Pi_{\phi}\right) f\left(\Pi_{\phi}\right),
$$

where $\Theta\left(X, \Pi_{\phi}\right)=g\left(\Pi_{\phi}\right) e^{i \Pi_{\phi} X}$ and the symbol * denotes complex conjugation. The Hilbert space of physical states is thus the space of wave functions of the form (10) with $f\left(\Pi_{\phi}\right) \in L^{2}\left(\mathbb{R}^{+}, d \Pi_{\phi} / \Pi_{\phi}\right)$. On the other hand, it is not difficult to check that the obtained representation is irreducible. 
Similar conclusions could have been reached if we had analysed instead the sector of negative momenta $\Pi_{\phi}$. In that case, one can choose as representation space the space of complex functions $\Psi\left(X, \Pi_{\phi}\right)$ on $\mathbb{R} \times \mathbb{R}^{-}$, with $\Pi_{\phi}<0$. The physical states are then the wave functions

$$
\Psi\left(X, \Pi_{\phi}\right)=\tilde{f}\left(\Pi_{\phi}\right) e^{-i \Pi_{\phi} X},
$$

with $\tilde{f}\left(\Pi_{\phi}\right)$ a generic function on $\mathbb{R}^{-}$. They satisfy the constraint

$$
\hat{\Pi} \Psi\left(X, \Pi_{\phi}\right)=-\Pi_{\phi} \Psi\left(X, \Pi_{\phi}\right) .
$$

The Lorentzian reality conditions determine now the inner product

$$
<\Theta, \Psi>=\int_{\mathbb{R}^{-}} \frac{d \Pi_{\phi}}{\left|\Pi_{\phi}\right|} \tilde{g}^{*}\left(\Pi_{\phi}\right) \tilde{f}\left(\Pi_{\phi}\right),
$$

where $\Theta\left(X, \Pi_{\phi}\right)=\tilde{g}\left(\Pi_{\phi}\right) e^{-i \Pi_{\phi} X}$. Thus, the Hilbert space consists of all functions (14) with $\tilde{f}\left(\Pi_{\phi}\right) \in L^{2}\left(\mathbb{R}^{-}, d \Pi_{\phi} /\left|\Pi_{\phi}\right|\right)$. It is then easy to show that the representations of the quantum theory constructed here for $\Pi_{\phi}>0$ and $\Pi_{\phi}<0$ are unitarily equivalent.

In the next section, we will translate these results into the geometrodynamic representation in which the wormhole wave functions of this model have been obtained [5-7]. In order to do that, it will be useful to introduce first a different set of elementary operators in the $\left(X, \Pi_{\phi}\right)$ representation that we have studied. The reason for this is that the action of these new elementary operators will have a direct counterpart in the geometrodynamic representation that we are going to analyse. Let us then define (either for $\Pi_{\phi}>0$ or $\Pi_{\phi}<0$ ) the operators

$$
\begin{gathered}
\hat{\Pi}_{\phi} \Psi=\Pi_{\phi} \Psi\left(X, \Pi_{\phi}\right), \widehat{\Pi_{\phi}} \phi \Psi=i\left(\Pi_{\phi} \partial_{\Pi_{\phi}}-\frac{1}{2}\right) \Psi\left(X, \Pi_{\phi}\right), \\
(\Pi \widehat{\tanh X}) \Psi=-i \partial_{X}\left(\tanh X \Psi\left(X, \Pi_{\phi}\right)\right),\left(\frac{\widehat{\Pi}}{\cosh X}\right) \Psi=-i \partial_{X}\left(\frac{\Psi\left(X, \Pi_{\phi}\right)}{\cosh X}\right),
\end{gathered}
$$

with only non-vanishing commutators given by

$$
\left[\widehat{\Pi_{\phi}} \phi, \hat{\Pi}_{\phi}\right]=i \hat{\Pi}_{\phi}, \quad\left[(\Pi \widehat{\tanh } X),\left(\frac{\widehat{\Pi}}{\cosh X}\right)\right]=i\left(\frac{\widehat{\Pi}}{\cosh X}\right),
$$


so that they form a closed algebra under commutation relations. These operators represent a complete set of elementary variables, as can be proved by employing relations (4) and recalling that, for $q>0$ and $\Pi_{\phi}>0$ (or $\Pi_{\phi}<0$ ), the phase space of the unconstrained theory is the cotangent bundle over $\left(\mathbb{R}^{+}\right)^{2}$ (or $\mathbb{R}^{+} \times \mathbb{R}^{-}$, respectively). Equation (17) ensures that the action of $\widehat{\Pi_{\phi} \phi}$ on the physical states (10) or (14) coincides with that derived from relation (11) (and (7-9) and (15)) with the following factor ordering

$$
\widehat{\Pi_{\phi}} \phi=\hat{w}-\hat{\Pi}_{\phi}^{2} \frac{1}{2}\left(\hat{X} \hat{\Pi}^{-1}+\hat{\Pi}^{-1} \hat{X}\right) .
$$

The definitions (18), on the other hand, can be obtained from equation (8) with a particular choice of operator ordering, i.e., with the derivative operator $\hat{\Pi}$ acting on the left of any factor depending on $X$. This ordering guarantees that the operator $\hat{\Pi}^{2}$ acts exactly as the sum of the squares of $(\Pi \widehat{\tanh } X)$ and $\left(\frac{\widehat{\Pi}}{\cosh X}\right)$. Hence, the quantum version of the Hamiltonian constraint (6) can be rewritten as

$$
\hat{H} \Psi\left(X, \Pi_{\phi}\right) \equiv \frac{1}{2}\left[(\Pi \widehat{\tanh X})^{2}+\left(\frac{\widehat{\Pi}}{\cosh X}\right)^{2}-\hat{\Pi}_{\phi}^{2}\right] \Psi\left(X, \Pi_{\phi}\right)=0 .
$$

\section{Change of Representation}

We are now in an adequate position to discuss the relation between the $\left(X, \Pi_{\phi}\right)$ representation, used to perform the non-perturbative quantization, and the geometrodynamic $(q, \phi)$ representation in which the wormhole wave functions of the model are known [5-7]. Before carrying out any explicit calculations, let us notice that in the $(q, \phi)$ representation studied in the literature $[5,7]$ the momenta $\Pi_{\phi}$ has been assumed to run over the whole real axis, instead of being restricted to have either positive or negative values. The situation is in fact similar to that which one encounters when analysing the quantum theory of a relativistic particle subject to a Klein-Gordon constraint. Such theory admits two equivalent irreducible representations, one for positive frequencies and the other for negatives ones. In the position representation, 
however, the wave functions are usually allowed to get contributions from both types of frequencies. The representation obtained is not longer irreduciblef, but given by the direct sum of two irreducible representations, that correspond, respectively, to the sectors of positive and negative frequencies. Extrapolating these results to our model, we expect the geometrodynamic $(q, \phi)$ representation to be provided by the direct sum of the irreducible representations constructed for $\Pi_{\phi}>0$ and $\Pi_{\phi}<0$ in section 2. Adding these representations, the physical states of the theory adopt the generic expression

$$
\Psi\left(X, \Pi_{\phi}\right)=\left\{\begin{array}{cc}
f\left(\Pi_{\phi}\right) e^{i \Pi_{\phi} X} & \Pi_{\phi}>0 \\
\tilde{f}\left(\Pi_{\phi}\right) e^{-i \Pi_{\phi} X} & \Pi_{\phi}<0
\end{array},\right.
$$

and the physical inner product can be taken as

$$
<\Theta, \Psi>=\int_{\mathbb{R}^{+}} \frac{d \Pi_{\phi}}{\Pi_{\phi}} g^{*}\left(\Pi_{\phi}\right) f\left(\Pi_{\phi}\right)+\int_{\mathbb{R}^{-}} \frac{d \Pi_{\phi}}{\left|\Pi_{\phi}\right|} \tilde{g}^{*}\left(\Pi_{\phi}\right) \tilde{f}\left(\Pi_{\phi}\right),
$$

where $\left(g\left(\Pi_{\phi}\right), \tilde{g}\left(\Pi_{\phi}\right)\right)$ are the functions that characterize the state $\Theta\left(X, \Pi_{\phi}\right)$. Note that, in defining this inner product, there actually exists an ambiguity of a positive factor which determines the relative weight of the contributions from the representations with positive and negative momenta $\Pi_{\phi}$. Since these representations are physically equivalent, we have chosen that factor to be equal to the unity.

The change to the $(q, \phi)$ representation must be given by an integral transform of the type [16]

$$
\begin{gathered}
\Psi(q, \phi)=\sigma\left(\Psi\left(X, \Pi_{\phi}\right)\right)=\int_{\mathbb{R}^{+}} d \Pi_{\phi} g_{1}^{+}\left(\Pi_{\phi}\right) e^{i \phi \Pi_{\phi}} \int_{\mathbb{R}} d X g_{2}^{+}(X) e^{i F(q, X)} f\left(\Pi_{\phi}\right) e^{i \Pi_{\phi} X}+ \\
\int_{\mathbb{R}^{-}} d \Pi_{\phi} g_{1}^{-}\left(\Pi_{\phi}\right) e^{i \phi \Pi_{\phi}} \int_{\mathbb{R}} d X g_{2}^{-}(X) e^{i F(q, X)} \tilde{f}\left(\Pi_{\phi}\right) e^{-i \Pi_{\phi} X} .
\end{gathered}
$$

Here, $\sigma$ denotes the change of representation, and $F(q, X)$ is the generating function (5). The integral transform in $X$ must provide the change to some intermediate $\left(q, \Pi_{\phi}\right)$ representation, both for $\Pi_{\phi}>0$ and $\Pi_{\phi}<0$. The generalized Fourier transform in $\Pi_{\phi}$ leads then to desired $(q, \phi)$ representation, obtained as the direct

\footnotetext{
${ }^{*}$ Assuming that we do not allow time reversal operations
} 
sum of two irreducible pieces. The functions $\left(g_{1}^{+}, g_{2}^{+}, g_{1}^{-}, g_{2}^{-}\right)$that appear in formula (24) can be fixed by requiring that, under the integral transform, the operators $(17,18)$, defined in the $\left(X, \Pi_{\phi}\right)$ representation, act on $\Psi(q, \phi)$ in the following way:

$$
\begin{gathered}
\sigma\left(\hat{\Pi}_{\phi} \Psi\left(X, \Pi_{\phi}\right)\right) \equiv \hat{\Pi}_{\phi} \Psi(q, \phi)=-i \partial_{\phi} \Psi(q, \phi), \\
\sigma\left(\left(\frac{\widehat{\Pi}}{\cosh X}\right) \Psi\left(X, \Pi_{\phi}\right)\right) \equiv \hat{q} \Psi(q, \phi)=q \Psi(q, \phi), \\
\sigma\left(-(\Pi \widehat{\tanh } X) \Psi\left(X, \Pi_{\phi}\right)\right) \equiv \widehat{q \Pi_{q}} \Psi(q, \phi)=-i q \partial_{q} \Psi(q, \phi), \\
\sigma\left(\widehat{\Pi_{\phi}} \phi \Psi\left(X, \Pi_{\phi}\right)\right) \equiv \widehat{\Pi}_{\phi} \phi \Psi(q, \phi)=-i\left(\phi \partial_{\phi}+\frac{1}{2}\right) \Psi(q, \phi) .
\end{gathered}
$$

In equations $(26,27)$ we have used relations (4) to define the quantum operators $\hat{q}$ and $\widehat{q \Pi}_{q}$. Equations $(25,26)$, on the other hand, reproduce the standard action of the operators $\hat{\Pi}$ and $\hat{q}$ in the $(q, \phi)$ representation. Condition $(27)$ guarantees then that, under the change of representation, the quantum Hamiltonian constraint (21) translates into

$$
\hat{H} \Psi(q, \phi) \equiv \frac{1}{2}\left(q^{2}-\left(q \partial_{q}\right)^{2}+\partial_{\phi}^{2}\right) \Psi(q, \phi)=0,
$$

which is exactly the Wheeler-DeWitt equation (associated with constraint (3)) that is solved by the known wormhole wave functions of the model [5-7]. Thus, equation $(27)$, together with $(25,26)$, leads precisely to the factor ordering that has been employed in the literature for the geometrodynamic analysis of this minisuperspace. Finally, requirement (28) allows us to interpret the action of $\widehat{\Pi_{\phi}} \phi$ in the $(q, \phi)$ representation as the symmetrized product of the multiplicative operator $\hat{\phi}$ (with $\hat{\phi} \Psi=\phi \Psi(q, \phi)$ ) and $\hat{\Pi}_{\phi}=-i \partial_{\phi}$.

A careful calculation shows then that, for all those physical states that are normalizable with respect to the inner product (23), the change of representation (24) is well-defined and satisfies conditions (25-28) if and only if $g_{2}^{+}$and $g_{2}^{-}$are nonvanishing constants and $g_{1}^{+}$and $g_{1}^{-}$are proportional to $\Pi_{\phi}^{-1}$. So, we can let in the 
following

$$
g_{1}^{+}=g_{1}^{-}=\frac{1}{\Pi_{\phi}}, \quad g_{2}^{+}=g_{2}^{-}=1 .
$$

Using now that, for $q>0[17]$

$$
\int_{\mathbb{R}} d X e^{-i q \sinh X+i \Pi_{\phi} X}=2 e^{\frac{\pi}{2} \Pi_{\phi}} K_{i \Pi_{\phi}}(q),
$$

with $K_{i \Pi_{\phi}}$ a modified Bessel function of imaginary order, equation (24) can be rewritten in the compact form

$$
\Psi(q, \phi)=\int_{\mathbb{R}^{+}} \frac{d \Pi_{\phi}}{\Pi_{\phi}} 2 e^{\frac{\pi}{2} \Pi_{\phi}} K_{i \Pi_{\phi}}(q)\left(e^{i \phi \Pi_{\phi}} f\left(\Pi_{\phi}\right)+e^{-i \phi \Pi_{\phi}} h\left(\Pi_{\phi}\right)\right),
$$

where we have introduced the notation

$$
h\left(\Pi_{\phi}\right)=-\tilde{f}\left(-\Pi_{\phi}\right), \quad \Pi_{\phi}>0 .
$$

Hence, $h\left(\Pi_{\phi}\right) \in L^{2}\left(\mathbb{R}^{+}, d \Pi_{\phi} / \Pi_{\phi}\right)$ for states $\Psi$ in the physical Hilbert space, because in that case $\tilde{f}\left(\Pi_{\phi}\right) \in L^{2}\left(\mathbb{R}^{-}, d \Pi_{\phi} /\left|\Pi_{\phi}\right|\right)$.

The transformation (32) can be inverted to recover the expression of the wave functions in the $\left(X, \Pi_{\phi}\right)$ representation. The inversion is given by the formulas

$$
\begin{gathered}
f\left(\Pi_{\phi}\right)=\int_{\mathbb{R}^{+}} d q G\left(\Pi_{\phi}, q\right) \int_{\mathbb{R}} d \phi e^{-i \phi \Pi_{\phi}} \Psi(q, \phi), \\
h\left(\Pi_{\phi}\right)=\int_{\mathbb{R}^{+}} d q G\left(\Pi_{\phi}, q\right) \int_{\mathbb{R}} d \phi e^{i \phi \Pi_{\phi}} \Psi(q, \phi), \\
G\left(\Pi_{\phi}, q\right)=\frac{2 \Pi_{\phi}}{\pi^{2}} e^{-\frac{\pi}{2} \Pi_{\phi}} \cosh \left(\pi \Pi_{\phi}\right) K_{i \Pi_{\phi}}(q) .
\end{gathered}
$$

In deriving these equations, we have employed that [18]

$$
\int_{0}^{\infty} d q K_{i \Pi_{\phi}}^{2}(q)=\frac{\pi^{2}}{4 \cosh \left(\pi \Pi_{\phi}\right)} .
$$

Substituting then (34-36) in (23), it is possible to derive the explicit form of the physical inner product in the geometrodynamic representation $(q, \phi)$.

Equation (32) implies in particular that, in the adopted $(q, \phi)$ representation, the Hilbert space of physical states is spanned by the functions

$$
\Psi_{\epsilon \tilde{\Pi}_{\phi}}(q, \phi)=\frac{2}{\sqrt{\tilde{\Pi}_{\phi}}} e^{\frac{\pi}{2} \tilde{\Pi}_{\phi}} K_{i \tilde{\Pi}_{\phi}}(q) e^{i \epsilon \phi \tilde{\Pi}_{\phi}}
$$


where $\epsilon=1$ or -1 and $\tilde{\Pi}_{\phi}$ is any positive constant $\left(\tilde{\Pi}_{\phi} \in \mathbb{R}^{+}\right)$. These functions are physical states of the model $[5,7]$, and can be obtained from the generic formula (32) by letting

$$
f\left(\Pi_{\phi}\right)=\sqrt{\Pi_{\phi}} \delta\left(\Pi_{\phi}-\tilde{\Pi}_{\phi}\right) \equiv f_{+\tilde{\Pi}_{\phi}}\left(\Pi_{\phi}\right), \quad h\left(\Pi_{\phi}\right)=0 \equiv h_{+\tilde{\Pi}_{\phi}}\left(\Pi_{\phi}\right)
$$

if $\epsilon=1$, or

$$
f\left(\Pi_{\phi}\right)=0 \equiv f_{-\tilde{\Pi}_{\phi}}\left(\Pi_{\phi}\right), \quad h\left(\Pi_{\phi}\right)=\sqrt{\Pi_{\phi}} \delta\left(\Pi_{\phi}-\tilde{\Pi}_{\phi}\right) \equiv h_{-\tilde{\Pi}_{\phi}}\left(\Pi_{\phi}\right)
$$

when $\epsilon=-1$. Recalling equation (33), it is then straightforward to check that these wave functions are not normalizable with respect to the inner product $(23)$. Nevertheless, they satisfy the orthogonality conditions

$$
<\Psi_{\epsilon^{\prime} \tilde{\Pi}_{\phi}^{\prime}}, \Psi_{\epsilon \tilde{\Pi}_{\phi}}>=\delta_{\epsilon^{\prime} \epsilon} \delta\left(\tilde{\Pi}_{\phi}^{\prime}-\tilde{\Pi}_{\phi}\right)
$$

with $\delta_{\epsilon^{\prime} \epsilon}$ the Kronecker delta. In this way, the set of wave functions (38) turns out to provide an orthogonal (but not normalizable) basis for the Hilbert space of the model in the $(q, \phi)$ representation.

The physical states (38) were first found by Hawking and Page when discussing the existence of quantum wormhole solutions in the considered minisuperspace [5]. To facilitate the comparison of our results with those of $[5,7]$, we note that the wave functions (38) with $\epsilon=-1$ can be equivalently rewritten as

$$
\Psi_{-\tilde{\Pi}_{\phi}}(q, \phi)=\tilde{\Psi}_{\tilde{\Pi}_{\phi}^{\prime}}(q, \phi)=\frac{2}{\sqrt{\left|\tilde{\Pi}_{\phi}^{\prime}\right|}} e^{\frac{\pi}{2}\left|\tilde{\Pi}_{\phi}^{\prime}\right|} K_{i \tilde{\Pi}_{\phi}^{\prime}}(q) e^{i \phi \tilde{\Pi}_{\phi}^{\prime}}
$$

where $\tilde{\Pi}_{\phi}^{\prime}=-\tilde{\Pi}_{\phi}<0$ and we have used that $K_{i \tilde{\Pi}_{\phi}}(q)=K_{-i \tilde{\Pi}_{\phi}}(q)$. Actually, the physical states (38) can be considered as generalized wormhole wave functions, in the sense that, even though they fail to fulfil the requirement of regularity when the three-geometry collapse to zero $(q \rightarrow 0)$, they are all exponentially damped for large scale factors $(q \rightarrow \infty)$ [5]. Thus, neglecting for the moment the regularity condition at $q=0$, we can assert that the space of states spanned in this model 
by the quantum wormhole solutions does not only admit a Hilbert structure, but coincides in addition with the Hilbert space of the Lorentzian theory. Moreover, in the next section we will show that, with a change of basis, it is possible to find a complete set of states that are genuine wormhole solutions and therefore do not display any singularity at the origin $q=0$.

The different wave functions (38) can be designated by the number $\epsilon \tilde{\Pi}_{\phi}$, which is the eigenvalue taken by the observable $\hat{\Pi}_{\phi}$ in these wormhole states. In this way, the wormhole parameter of the model $\left(\epsilon \tilde{\Pi}_{\phi}\right)$ turns out to be the eigenvalue of a quantum observable, in agreement with our comments in the Introduction.

Finally, equation (32) allows us to express the physical states in the $(q, \phi)$ representation as the following superposition of wormhole wave functions:

$$
\Psi(q, \phi)=\int_{\mathbb{R}^{+}} d \Pi_{\phi}\left(\frac{f\left(\Pi_{\phi}\right)}{\sqrt{\Pi_{\phi}}} \Psi_{+\Pi_{\phi}}(q, \phi)+\frac{h\left(\Pi_{\phi}\right)}{\sqrt{\Pi_{\phi}}} \Psi_{-\Pi_{\phi}}(q, \phi)\right) .
$$

The functions $f\left(\Pi_{\phi}\right)$ and $h\left(\Pi_{\phi}\right)$ that describe the quantum states in the $\left(X, \Pi_{\phi}\right)$

representation provide then, when divided by $\sqrt{\Pi_{\phi}}$, the respective contributions of the wormhole solutions $\Psi_{+\Pi_{\phi}}$ and $\Psi_{-\Pi_{\phi}}$ to the physical state $\Psi(q, \phi)$.

\section{Bases of Normalizable Wormholes}

In the previous section we have proved that the Lorentzian reality conditions select a well-defined inner product in the $(q, \phi)$ representation, and that the Hilbert space determined by that inner product is spanned by a set of generalized wormhole solutions. We want to show now that it is possible to find other bases for the Hilbert space of the model such that their elements represent proper wormhole states which are regular everywhere in the configuration space $\left(q \in \mathbb{R}^{+}, \phi \in \mathbb{R}\right)$ and decrease exponentially for large scale factors $q \gg 1$. In addition, the wormhole wave functions that form the bases to be considered will be seen to be normalizable with respect to the Lorentzian inner product (23). 
Let us study first the set of wormhole solutions given by

$$
\Psi_{\phi_{0}}(q, \phi)=e^{-q \cosh \left(\phi-\phi_{0}\right)}
$$

where $\phi_{0} \in \mathbb{R}$. These states can be obtained as path integrals over asymptotically flat manifolds and over matter fields that take the value $\phi_{0}$ in the asymptotic region of large three-volume [6]. These wave functions are clearly regular when the threegeometry collapses to zero $(q \rightarrow 0)$ and exponentially damped for $q \rightarrow \infty$. Thus, they represent genuine wormhole solutions.

The physical states (44) can be recovered from equation (32) with the choice

$$
\begin{gathered}
f\left(\Pi_{\phi}\right)=\frac{1}{2 \pi} e^{-i \Pi_{\phi} \phi_{0}} e^{-\frac{\pi}{2} \Pi_{\phi}} \Pi_{\phi} \equiv f_{\phi_{0}}\left(\Pi_{\phi}\right), \\
h\left(\Pi_{\phi}\right) \equiv h_{\phi_{0}}\left(\Pi_{\phi}\right)=f_{\phi_{0}}^{*}\left(\Pi_{\phi}\right),
\end{gathered}
$$

as can be easily checked by taking into account that [18]

$$
\int_{\mathbb{R}} d p e^{i p\left(\phi-\phi_{0}\right)} K_{i p}(q)=\pi e^{-q \cosh \left(\phi-\phi_{0}\right)}, \quad q>0
$$

From relation (47) it also follows that the generalized wormholes (38) can be expressed as superpositions of the wave functions $\Psi_{\phi_{0}}(q, \phi)$ :

$$
\Psi_{\epsilon \tilde{\Pi}_{\phi}}(q, \phi)=\frac{2}{\sqrt{\tilde{\Pi}_{\phi}}} e^{\frac{\pi}{2} \tilde{\Pi}_{\phi}} \int_{\mathbb{R}} d\left(\phi-\phi_{0}\right) e^{i \epsilon \tilde{\Pi}_{\phi} \phi_{0}} \Psi_{\phi_{0}}(q, \phi) .
$$

The set of wave functions $\Psi_{\epsilon \tilde{\Pi}_{\phi}}$ and $\Psi_{\phi_{0}}$ result then in spanning the same space of physical states. Therefore, the wormhole solutions (44) provide a basis for the Hilbert space of the model in the $(q, \phi)$ representation. Equation (48) can then be interpreted as the formula for the change of basis from the set $\Psi_{\epsilon \tilde{\Pi}_{\phi}}\left(\epsilon \tilde{\Pi}_{\phi} \in \mathbb{R}\right)$ to the new set $\Psi_{\phi_{0}}\left(\phi_{0} \in \mathbb{R}\right)$.

The wormhole wave functions (44) are completely characterized by the value of the parameter $\phi_{0}$. Using equations $(22),(33)$ and $(45,46)$ to describe these wormhole states in the $\left(X, \Pi_{\phi}\right)$ representation, it is not difficult to check that the wormhole 
parameter $\phi_{0}$ coincides in fact with the eigenvalue reached by the observable

$$
\left(e^{-\frac{\pi}{2}\left|\hat{\Pi}_{\phi}\right|} \hat{w} e^{\frac{\pi}{2}\left|\hat{\Pi}_{\phi}\right|} \hat{\Pi}_{\phi}^{-1}\right)
$$

in the considered wormhole solutions, with $\hat{\Pi}_{\phi}$ and $\hat{w}$ defined, respectively, by (7) and (11) (both for $\Pi_{\phi}>0$ and $\Pi_{\phi}<0$ ).

Finally, from equations $(33)$ and $(45,46)$ it is straightforward to prove that the wormhole wave functions (44) are normalizable with respect to the inner product (23), so that they all represent proper physical states of the quantum theory.

Besides the normalizable basis (44), there exists another known complete set of wormhole solutions for the model that we are studying $[5,7]$. The elements of this set adopt the expression

$$
\Psi_{n}(q, \phi)=\frac{1}{2^{n} n ! \sqrt{\pi}} H_{n}\left(\sqrt{2 q} \cosh \frac{\phi}{2}\right) H_{n}\left(\sqrt{2 q} \sinh \frac{\phi}{2}\right) e^{-q \cosh \phi} .
$$

Here, $n$ is a non-negative integer $(n=0,1, \ldots)$ and $H_{n}$ are the Hermite polynomials [18]. These wave functions vanish exponentially when $q \rightarrow \infty$, present no singularities in the region $\{q>0, \phi \in \mathbb{R}\}$ and possess a finite limit when $q$ tends to zero. In this sense, they can all be considered as truly quantum wormholes. In addition, they form a discrete basis for the Hilbert space of the system, since the wave functions (38) can be written as the following combinations of the wormholes $\Psi_{n}[7]$ :

$$
\begin{gathered}
\Psi_{\epsilon \tilde{\Pi}_{\phi}}(q, \phi)=\sum_{n=0}^{\infty} F_{n}\left(\epsilon \tilde{\Pi}_{\phi}\right) \Psi_{n}(q, \phi), \\
F_{n}\left(\epsilon \tilde{\Pi}_{\phi}\right)=2 \sqrt{\frac{\pi}{\tilde{\Pi}_{\phi}}} e^{\frac{\pi}{2} \tilde{\Pi}_{\phi}} \int_{\mathbb{R}} d \eta \frac{\sinh ^{n} \eta}{\cosh ^{n+1} \eta} e^{i 2 \eta \epsilon \tilde{\Pi}_{\phi}} .
\end{gathered}
$$

The wormhole states (50) are eigenstates of the operators

$$
\frac{1}{2}\left(x^{2}-\partial_{x}^{2}\right) \text { and } \frac{1}{2}\left(y^{2}-\partial_{y}^{2}\right)
$$

with eigenvalues equal to $n+\frac{1}{2}$, where $[5,7]$

$$
x=\sqrt{2 q} \cosh \frac{\phi}{2}, \quad y=\sqrt{2 q} \sinh \frac{\phi}{2} .
$$


We then expect the operators (53) to be observables of the model, for the integer $n$ corresponds to a well-defined wormhole parameter. That this is indeed the case can be proved by realizing that, from relations (54), one can rewrite the quantum Hamiltonian constraint of the system (29) as

$$
\hat{H} \equiv 8\left(x^{2}-y^{2}\right)\left(x^{2}-y^{2}-\partial_{x}^{2}+\partial_{y}^{2}\right)=0 .
$$

The operators (53) commute weakly with the above Hamiltonian, because, for $q>0$, equations (54) imply that $x$ is always greater than $|y|$.

On the other hand, using formulas (34-36) one can find the explicit expression of the wormhole wave functions $(50)$ in the $\left(X, \Pi_{\phi}\right)$ representation. A careful calculation leads to the result:

$$
\begin{gathered}
f\left(\Pi_{\phi}\right)=\frac{1}{\pi \sqrt{\pi}} \cosh \left(\pi \Pi_{\phi}\right) e^{-\frac{\pi}{2} \Pi_{\phi}} \Pi_{\phi} \int_{\mathbb{R}} d \eta \frac{\sinh ^{n} \eta}{\cosh ^{n+1} \eta} e^{-i 2 \eta \Pi_{\phi}} \equiv f_{n}\left(\Pi_{\phi}\right), \\
h\left(\Pi_{\phi}\right) \equiv h_{n}\left(\Pi_{\phi}\right)=(-1)^{n} f_{n}\left(\Pi_{\phi}\right) .
\end{gathered}
$$

The wave functions $\Psi_{n}\left(X, \Pi_{\phi}\right)$ can then be obtained from these equations and relations (22) and (33). In particular, the wormhole states (50) turn out to be normalizable with respect to the inner product (23), because the functions $\left(f_{n}\left(\Pi_{\phi}\right), h_{n}\left(\Pi_{\phi}\right)\right)$ are analytic for all positive $\Pi_{\phi}$, vanish when $\Pi_{\phi}$ tends to zero and decrease at infinity at least as fast as $\Pi_{\phi}^{-1}$, for it can be proved that

$$
\left|\lim _{\Pi_{\phi} \rightarrow \infty} e^{\frac{\pi}{2} \Pi_{\phi}} \Pi_{\phi}^{2} \int_{\mathbb{R}} d \eta \frac{\sinh ^{n} \eta}{\cosh ^{n+1} \eta} e^{-i 2 \eta \Pi_{\phi}}\right|<\infty .
$$

Therefore, the wave functions (50) represent proper physical states of the quantum theory. Finally, let us notice that the Hilbert space of the model is separable, because the basis (50) is countable and normalizable. As an additional consequence, the basis of wormholes $\Psi_{n}(n=0,1, \ldots)$ can then be orthonormalized using the standard Gram-Schmidt method. 


\section{Conclusions}

We have argued that if for a given representation the space of physical states spanned by the wormhole wave functions can be equipped with a Hilbert structure, that space must coincide with the Hilbert space of the Lorentzian gravitational system under consideration. According to Ashtekar's proposal $[9,10]$, the inner product in that space can then be determined by imposing a set of Lorentzian reality conditions. The wormhole states supply us in this way with a basis for the physical Hilbert space of the Lorentzian quantum theory. The elements of that basis are designated by a set of wormhole parameters which, in order to be well-defined quantum numbers that characterize the wormhole states, have to coincide with the eigenvalues reached in the wormhole wave functions by a complete set of compatible observables. In fact, the spectra of those observables must be equal to the ranges of the wormhole parameters for the wormhole solutions to form a complete set of physical states.

We have proved the correctness of the above statements in the particular case of a Friedmann-Robertson-Walker spacetime minimally coupled to a massless scalar field. For this minisuperspace, the wormhole wave functions were already known in the literature [5-7]. We have found the inner product selected by the Lorentzian reality conditions, and shown that the Hilbert space of the model admits a basis of normalizable wormhole states. We have considered in detail two normalizable bases of wormholes, one of them given by an infinite but countable number of wormhole solutions. The elements of any of these two bases turn out to be eigenfunctions of a certain observable of the system, and the corresponding eigenvalue provides the parameter that describes the different wormholes. We notice that, in this case, the eigenvalue of one observable is enough to characterize a quantum state, because the minisuperspace possesses only one physical degree of freedom. On the other hand, from the existence of a discrete basis of normalizable wormholes, it follows that the 
Hilbert space of the model is separable and admits an orthonormal basis.

Actually, a similar analysis to that presented here can also be carried out in the two other minisuperspace models in which a complete set of wormhole wave functions has already been obtained, i.e., a Friedmann-Robertson-Walker spacetime conformally coupled to a massless scalar field [7] and a Kantowski-Sachs spacetime provided with a minimally coupled scalar field with no mass [8]. In those models, the space of wormholes can be supplied as well with a Hilbert structure, and the corresponding Hilbert space can also be seen to coincide with the Hilbert space of the Lorentzian quantum theory. The explicit results for these minisuperspaces will be published elsewhere [19].

If there always exists a basis of wormhole wave functions for the Hilbert space of Lorentzian gravity, every quantum gravitational state can be interpreted as a superposition of wormholes. The amplitudes of the contributions of the different wormhole solutions in those superpositions provide a complete description of the proper physical states of the theory. On the other hand, if the quantum state of the Universe can be represented as a normalizable wave function, such a wave function must then be given by a particular combination of wormholes. This implies, in particular, that the wave function of the Universe must be damped for large threegeometries. Whatever the requirements to determine the quantum state of the Universe may be, they should guarantee this asymptotic behaviour. One should then expect the kind of boundary conditions usually employed in quantum cosmology (i.e., the no-boundary proposal [20] and the "tunneling" proposals of Linde [21] and of Vilenkin [22]) not to pick out, in general, a normalizable wave function to represent the Universe, since these conditions do not ensure the vanishing of the wave function for asymptotic large three-geometries. Therefore, these boundary conditions must be either modified or replaced in order to select a proper physical state that can describe our Universe. Let us finally comment that, if the wormhole 
solutions can be defined in full gravity as Euclidean path integrals [6], it must be possible to obtain the wave function of the Universe as a particular sum over histories on manifolds that possess an asymptotic region of large three-volume where there are no gravitational excitations. In this sense, the asymptotic flatness of our Universe would be a prediction of the quantum theory of gravity.

\section{Acknowledgements}

The author wants to thank P. González Díaz, J. Mourao and C. Soo for helpful conversations. This work was supported by funds provided by the Spanish Ministry of Education and Science Grant No. EX92-06996911. 


\section{References}

[1] Hawking S W 1988 Phys. Rev. D 37904

[2] Giddings S and Strominger A 1988 Nucl. Phys. B 306890

Hosoya A and Ogura W 1989 Phys. Lett. B 225117

Halliwell J J and Laflamme R 1989 Class. Quantum Grav. 61839

[3] Coleman S 1988 Nucl. Phys. B 310643

Hawking S W 1990 Nucl. Phys. B 335155

[4] Bochner S 1946 Bull. Am. Math. Soc. 52776

Cheeger J and Gromoll D 1972 Ann. Math. 96413

[5] Hawking S W and Page D N 1990 Phys. Rev. D 422655

[6] Garay L J 1991 Phys. Rev. D 441059

[7] Garay L J 1993 Phys. Rev. D 481710

[8] Campbell L M and Garay L J 1991 Phys. Lett. B 25449

[9] Ashtekar A 1991 Lectures on Non-Perturbative Canonical Gravity ed Fang L Z and Ruffini R (Singapore: World Scientific)

[10] Ashtekar A 1993 Gravitation and Quantizations, Les Houches Summer School Proceedings Vol. LVII ed Julia B and Zinn-Justin J (Amsterdam: North Holland)

[11] Ashtekar A 1986 Phys. Rev. Lett. 57 2244; 1987 Phys. Rev. D 361587

[12] Rendall A 1993 Class. Quantum Grav. 102261

[13] Ashtekar A, Romano J D and Tate R S 1989 Phys. Rev. D 402572 
[14] Mena Marugán G A 1993 Reality Conditions for Lorentzian and Euclidean Gravity in the Ashtekar Formulation, Preprint Penn State University CGPG93/11-2, to appear in Int. J. Mod. Phys. D

[15] Ashtekar A, Tate R and Uggla C 1993 Int. J. Mod. Phys. D 215

[16] Mena Marugán G A 1993 Reality Conditions in Non-Perturbative Quantum Cosmology, Preprint Penn State University CGPG-93/9-3, to appear in Class. Quantum Grav.

[17] Abramowitz M and Stegun I A (ed) 1970 Handbook of Mathematical Functions 9th edn (Natl. Bur. Stand. Appl. Math. Ser. No. 55) (Washington, D.C.: U.S. Govt. Print. Off.)

[18] Gradshteyn I S and Ryzhik I M 1980 Table of Integrals, Series and Products 4th edn (San Diego: Academic Press)

[19] Mena Marugán G A 1994 in preparation

[20] Hawking S W 1982 Astrophysical Cosmology ed Brück H A, Coyne G V and Longair M S (Vatican City: Pontificia Academia Scientarium); 1984 Nucl. Phys. B 239257

Hartle J B and Hawking S W 1983 Phys. Rev. D 282960

[21] Linde A 1984 Zh. Eksp. Teor. Fiz. 87369 (1984 Sov. Phys. JETP 60 211); 1984 Nuovo Cimento 39 401; 1984 Rep. Prog. Phys. 47925

[22] Vilenkin A 1984 Phys. Rev. D 30 509; 1986 Phys. Rev. D 33 3560; 1988 Phys. Rev. D 37888 